\title{
AREA-PERIMETER DUALITY IN POLYGON SPACES
}

\author{
GIORGI KHIMSHIASHVILI, GAIANE PANINA, DIRK SIERSMA
}

\begin{abstract}
Two natural foliations, guided by area and perimeter, of the configurations spaces of planar polygons are considered and the topology of their leaves is investigated in some detail. In particular, the homology groups and the homotopy type of leaves are determined. The homology groups of the spaces of polygons with fixed area and perimeter are also determined. Besides, we extend the classical isoperimetric duality to all critical points. In conclusion a few general remarks on dual extremal problems in polygon spaces and beyond are given.
\end{abstract}

\section{INTRODUCTION}

The aim of the present paper is to explicate and generalize the results of the classical isoperimetric problem in polygon spaces given in [2, 7]. In particular, we complement results of [7] on the critical points of area on the space of polygons with fixed perimeter and generalize the results of the structure of area foliation in the space of triangles given in [5]. One of the essential novel ingredients of our approach is a conceptual generalization of the well-known duality between area and perimeter in the classical isoperimetric problem (see e.g. [8]). Namely, we extend the idea of duality to all critical points of the two dual extremal problems in question. Technically, this involves comparison of the Morse indices of two smooth functions at a generic tangent point of their level surfaces and applying a type of argument in the spirit of 'rabattement de diagramme de Cerf' widely used in singularity theory.

This, in particular, enables us to show that the perimeter function on the space of polygons with constant non-zero area is level-preserving homeomorphic to the area function on the space of polygon with constant perimeter and positive area (Theorem 3), which may be considered as one of the main results of the present paper. We describe also topological structure of these spaces.

Another new result (which is crucial for the whole paper) is determining the homology groups of the spaces of $n$-gons with both area and perimeter fixed (Theorem 21).

\section{Preliminaries}

An $n$-gon is an $n$-tuple of points $\left(p_{1}, \ldots, p_{n}\right) \in\left(\mathbb{R}^{2}\right)^{n}$ modulo diagonal action of orientation preserving isometries of the plane. Some (but not all) of $p_{i}$ may coincide.

The space $\mathcal{C}=\mathcal{C}^{n}$ of all $n$-gons for a fixed $n$ is equipped by two natural functions

$$
\mathcal{A}, \mathcal{P}: \mathcal{C} \rightrightarrows \mathbb{R}:
$$

2000 Mathematics Subject Classification. 52R70, 52B99.

Key words and phrases. Morse index, critical point, Morse surgery, configuration space. 
- The perimeter of a polygon is (as usual)

$$
\mathcal{P}\left(p_{1}, \ldots, p_{n}\right)=\left|p_{1} p_{2}\right|+\left|p_{2} p_{3}\right|+\ldots+\left|p_{n} p_{1}\right| .
$$

- The oriented area is defined as

$$
2 \mathcal{A}\left(p_{1}, \ldots, p_{n}\right)=x_{1} y_{2}-x_{2} y_{1}+\ldots+x_{n} y_{1}-x_{1} y_{n},
$$

where $p_{i}=\left(x_{i}, y_{i}\right)$.

One sees immediately that perimeter of a polygon is always positive, whereas the area can be also zero or negative. In particular, the set $\{\mathcal{A}=0\}$ splits $\mathcal{C}$ into two mutually symmetric parts.

Oriented area as a Morse function has been studied in various settings: for configuration spaces of flexible polygons (those with side lengths fixed), see [4, 9], [13, [14]; for the space with perimeter fixed [7] and with other constraints. Probably the most complete case has been treated in [11].

Polygons with fixed perimeter [7],[11]. We shall need the following result:

Let $\sigma$ be a cyclic renumbering: given a polygon $P=\left(p_{1}, \ldots, p_{n}\right)$,

$$
\sigma\left(p_{1}, \ldots, p_{n}\right)=\left(p_{2}, p_{3}, \ldots, p_{n}, p_{1}\right) .
$$

In other words, we have an action of $\mathbb{Z}_{n}$ on $\mathcal{C}$ which renumbers the vertices of a polygon cyclically. A regular star is an equilateral $n$-gon such that $\sigma\left(p_{1}, \ldots, p_{n}\right)=$ $\left(p_{1}, \ldots, p_{n}\right)$, see Figure 1

A complete fold is a regular star with $p_{i}=p_{i+2}$. It exists for even $n$ only.

A regular star $P$ which is not a complete fold is uniquely defined by its winding number $w(P)$ with respect to the center.

Let $\mathcal{C}_{\pi}$ be the space of all $n$-gons with some fixed value $\pi>0$ of perimeter.
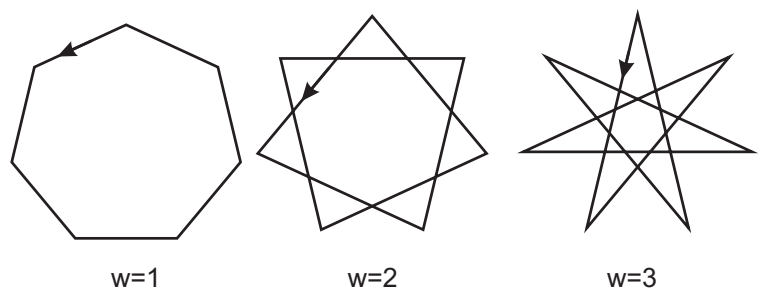

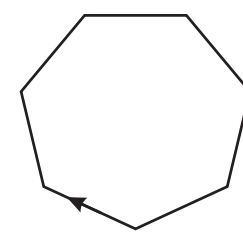

$w=-1$

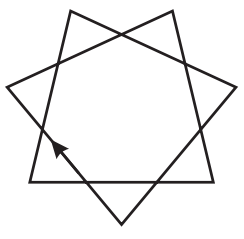

$w=-2$

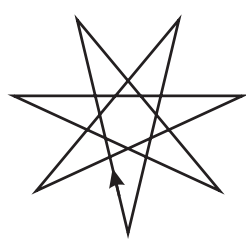

$w=-3$

Figure 1. Regular stars for $n=7$ with their winding numbers. 
Theorem 1. [7, 11

(1) $\mathcal{C}_{\pi}$ is homeomorphic to $\mathbb{C} P^{n-2}$.

(2) The restriction $\left.\mathcal{A}\right|_{\mathcal{C}_{\pi}}$ is a perfect Morse function.

(3) The critical points of the function $\left.\mathcal{A}\right|_{\mathcal{C}_{\pi}}$ are regular stars and complete folds only.

(4) The Morse index of a regular star is:

$$
M(P)= \begin{cases}2 w(P)-2, & \text { if } w(P)<0 ; \\ 2 n-2 w(P)-2, & \text { if } w(P)>0 ; \\ n-2, & \text { if } P \text { is a complete fold (for even } n \text { only). }\end{cases}
$$

(5) The critical values of function $\left.\mathcal{A}\right|_{\mathcal{C}_{\pi}}$ increase with the Morse index.

The theorem was proved in [7] under assumption that the critical points are nondegenerate Morse points. Later on, it was proven in [11] that the the critical points are indeed non-degenerate. The proof was missing for complete folds, but now we provide the proof in the Appendix.

It follows from (1) that the space $\mathcal{C}$ of all $n$-gons can be viewed as a cylinder over $\mathbb{C} P^{n-2}$, or rather as a cone over $\mathbb{C} P^{n-2}$ with the apex removed.

Polygons with fixed area. Denote by $\mathcal{C}_{a}$ the space of polygons with fixed value $a$ of $\mathcal{A}$. (We distinguish between $\mathcal{C}_{a}$ and $\mathcal{C}_{\pi}$ by arabic and greek letters.) By scaling, it is clear that all $\mathcal{C}_{a}$ with $a \neq 0$ are homeomorphic. In Theorems 4 and 5 we state analogues of Theorem 1 for $\left.\mathcal{P}\right|_{\mathcal{C}_{a}}$.

Area and perimeter in interaction. The space $\mathcal{C}$ has two foliations: one by level sets of the perimeter, and the other one by level sets of the oriented area. Area can be considered as a function on the leaves of the perimeter foliation, and vice versa.

One of the main goals of the paper is to compare the level foliations of $\mathcal{A}$ and $\mathcal{P}$. Let us first mention that the minimum of $\left.\mathcal{P}\right|_{\mathcal{C}_{a}}$ corresponds to the maximum of $\left.\mathcal{A}\right|_{\mathcal{C}_{\pi}}$. This is called the 'isoperimetric duality'. To extend it to all critical points and level surfaces, let us take together area and perimeter. We obtain a map

$$
\begin{gathered}
\Phi: \mathcal{C} \rightarrow \mathbb{R}_{>0} \times \mathbb{R}, \\
\Phi(P)=(\mathcal{P}(P), \mathcal{A}(P)) .
\end{gathered}
$$

Proposition 1. (1) The critical set $\Gamma \subset \mathcal{C}$ of $\Phi$ consists of finitely many curves.

(2) The discriminant set $D \subset \mathbb{R}_{>0} \times \mathbb{R}$ of $\Phi$ is a finite set of curves $D_{i}$ given by $\mathcal{A}=c_{i} \mathcal{P}^{2}$. The curves $D_{i}$ correspond to regular stars and complete folds.

(3) $\Phi$ is a locally trivial fiber bundle over $\mathbb{R}_{>0} \times \mathbb{R} \backslash D$.

(4) The regular fibers are submanifolds of $\mathcal{C}$ of codimension 2. Each singular fiber has a Morse-type singularity. 
Proof. If a polygon $P$ is critical for $\Phi$, then so is any dilate of $P$. Due to homogeneity of $\mathcal{A}$ and $\mathcal{P}$, the map $\Phi$ sends each line $c P$ to a curve $A=c P^{2}$ in $\mathbb{R}_{>0} \times \mathbb{R}$. The singularities of $\mathcal{A}$ on $\mathcal{C}_{\pi}$ are isolated and of Morse type, see Theorem 1. Therefore $\Gamma$ is one-dimensional, and the fibres above points of $D$ are of Morse type. The index is defined for every transversal slice, and due to homogeneity property, the index is constant for each curve.

To summarize, one can envisage the situation as follows. The image of $\Phi$ is the shadowed area in Figure 2.
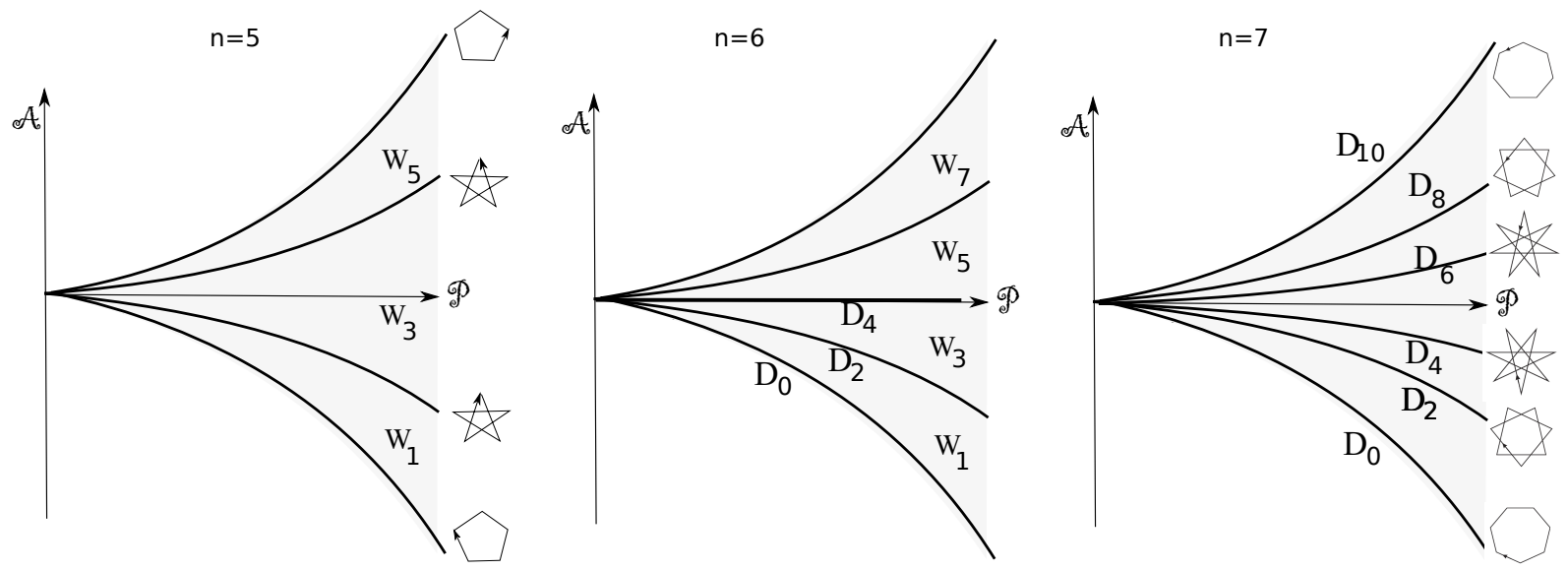

Figure 2. Stratification of $\mathbb{R}_{>0} \times \mathbb{R}$.

The bold curves $D_{i}$ are the images of the critical points. We number them accordingly to the Morse indices. For even and odd $n$ we have two different pictures: if $n$ is even, there appears a horizontal bold ray which corresponds to the complete folds. Each vertical straight line is the image of the space $\mathcal{C}_{\pi}$ for some $\pi$, and its intersection with a bold curve corresponds to a critical value described in Theorem 1.

The bold curves split the image of $\Phi$ into open domains; let us enumerate them starting from the bottom by odd numbers: $W_{1}, W_{3}, W_{5}$, etc. In each domain we have the same fiber type. Together with singular fibers, they constitute a codimension 2 foliation over $\mathcal{C}$ which is fiber homeomorphic to a product of $\mathbb{R}_{>0}$ (foliated by points) and the $\mathcal{A}$-foliation on $\mathcal{C}_{\pi}$.

These constructions and concepts are known as polar curves and Cerf diagrams in (complex) singularity theory, see e.g. [12].

\section{Configuration spaces of polygons With area And Perimeter fixed.}

For a point $(\pi, a) \in \mathbb{R}_{>0} \times \mathbb{R}$ set $\mathcal{C}_{\pi, a}=\Phi^{-1}(\pi, a)$

Theorem 2. (1) Let $i \leqslant n-2$. Assume that $(\pi, a) \in W_{i}$. The fiber $\mathcal{C}_{\pi, a}$ over this point is an $2 n-5$-dimensional non-singular manifold whose topological type depends on the domain $W_{i}$ only. The homology groups of $\mathcal{C}_{\pi, a}$ are: 


$$
H_{j}\left(\mathcal{C}_{\pi, a}, \mathbb{Z}\right)= \begin{cases}\mathbb{Z}, & \text { if } j \text { is even and } j \leqslant i ; \\ \mathbb{Z}, & \text { if } j \text { is odd and } 2 n-5 \geqslant j \geqslant 2 n-5-i \\ 0, & \text { otherwise. }\end{cases}
$$

(2) For $(\pi, a) \in D_{0}$, the space $\mathcal{C}_{\pi, a}$ is a single point. Let $0<i<n-2$, and $(\pi, a) \in D_{i}$. Then $\mathcal{C}_{\pi, a}$ is an $2 n-5$-dimensional manifold with one singular point. Its homology groups are:

$$
H_{j}\left(\mathcal{C}_{\pi, a}, \mathbb{Z}\right)= \begin{cases}\mathbb{Z}, & \text { if } j \text { is even and } j \leqslant i ; \\ \mathbb{Z}, & \text { if } j \text { is odd and } 2 n-5 \geqslant j \geqslant 2 n-5-i \\ 0, & \text { otherwise. }\end{cases}
$$

(3) If $i>n-2$, then the fiber $\mathcal{C}_{\pi, a}$ of a point $(\pi, a) \in W_{i}$ (respectively, of a point $\left.(\pi, a) \in D_{i}\right)$ is homeomorphic to the fiber over a point from $W_{2 n-i-4}$ (respectively, from $D_{2 n-i-4}$ ).

Their homology groups follow from (1) and (2).

(4) $\mathcal{C}_{\pi, 0}$ is an $2 n-5$-dimensional manifold (with a singularity if $n$ is even). Its homology groups are:

$$
H_{j}\left(\mathcal{C}_{\pi, 0}, \mathbb{Z}\right)= \begin{cases}\mathbb{Z}, & \text { if } j \text { is even and } j \leqslant n-2 ; \\ \mathbb{Z}, & \text { if } j \text { is odd and } 2 n-5 \geqslant j>n-2 \\ 0, & \text { otherwise. }\end{cases}
$$

Proof.

(3) is true since symmetry with respect a line keeps perimeter and takes $\mathcal{A}$ to $-\mathcal{A}$. Let us prove (1). Consider the restriction $\left.\mathcal{A}\right|_{\mathcal{C}_{\pi}}$. Set $A=\Phi^{-1}(\pi,(-\infty, a])$. By Morse theory, it has the homotopy type of a cell complex with even-dimensional cells only, one cell in each dimension starting from 0 to $i-1$.

Let us explain this in more details. Morse theory suggest to control the behaviour of $A=\left.\mathcal{A}\right|_{\mathcal{C}_{\pi}} ^{-1}(-\infty, x]$ and $\mathcal{A}^{-1}(x)$ as $x$ grows. When $x$ is very small, both preimages are empty. The space $A$ becomes a ball after passing through the minimum of $\left.\mathcal{A}\right|_{\mathcal{C}_{\pi}}$, whereas $\left.\mathcal{A}\right|_{\mathcal{C}_{\pi}} ^{-1}(x)$ turns to a sphere. For a regular value $x$ is a non-singular manifold. Passing through a critical value means (a) attaching a cell to $A$ whose dimension equals the Morse index and (b) applying a Morse surgery to $\left.\mathcal{A}\right|_{\mathcal{C}_{\pi}} ^{-1}(x)$. In our case all the indices are even.

An important feature of our particular case is that the function $\left.\mathcal{A}\right|_{\mathcal{C}_{\pi}}$ is not everywhere continuously differentiable, since the perimeter involves square roots. This problem has been treated in [7]. In short, Clarke subdifferential from nonsmooth analysis generalizes smooth Morse theory and provides the "regular interval" theorem which states, that (1) preimage of a Lipshitz-regular value is a manifold, and (2) if an interval contains no critical values, then the preimages of its endpoints are homeomorphic.

In [7] we checked that at each non-smooth point $\left.\mathcal{A}\right|_{\mathcal{C}_{\pi}}$ is regular, see Theorem 1. 
Analogously, the set $B=\Phi^{-1}(\pi,[a, \infty))$ has the homotopy type of a cell complex with even-dimensional cells only, one cell in each dimension starting from 0 to $2 n-$ $i-5$.

The union of $A$ and $B$ is homeomorphic to $\mathbb{C} P^{n-2}$ (Theorem 1), and the intersection is exactly the space $\mathcal{C}_{\pi, a}$ we are interested in. The Mayer-Vietoris exact sequence for these spaces can be visualized as a table, where the rows correspond to the indices of homology groups, and whose entries are homology groups of the participants of the sequence. The table below depicts the case $n=7$, and $(\pi, a) \in W_{3}$. Initially, the first column contains unknown homology groups. Let us show that they are recovered uniquely.

Lemma 1. Let $\mathbf{i}^{A}: A \subset A \cup B$ and $\mathbf{i}^{B}: B \subset A \cup B$ be inclusions. Then the induced homomorphisms

$$
\mathbf{i}_{*}^{A}: H_{*}(A, \mathbb{Z}) \rightarrow H_{*}(A \cup B, \mathbb{Z}) \text { and } \mathbf{i}_{*}^{B}: H_{*}(B, \mathbb{Z}) \rightarrow H_{*}(A \cup B, \mathbb{Z})
$$

are monomorphisms.

Proof follows from the cell complex structures of $A, B$, and $A \cup B$ : up to dimension $i$, the complex $A \cup B$ has exactly the same cells as $A$.

Depending on the index $i$, the Mayer-Vietoris sequence splits into three types of sequences:

(1) In the upper part, for $j=2 k, 2 n-5-i \leqslant j \leqslant 2 n-4$, we have

$$
0 \rightarrow H_{2 k}(A \cap B) \rightarrow 0 \rightarrow \mathbb{Z} \rightarrow H_{2 k-1}(A \cap B) \rightarrow 0
$$

One concludes that $H_{2 k}(A \cap B)=0, \quad H_{2 k-1}(A \cap B)=\mathbb{Z}$.

(2) Next comes $j=2 k, \quad i \leqslant j \leqslant 2 n-5-i$.

$$
0 \rightarrow H_{2 k}(A \cap B) \rightarrow \mathbb{Z} \rightarrow \mathbb{Z} \rightarrow H_{2 k-1}(A \cap B) \rightarrow 0
$$

By Lemma 1 the central arrow is the identity map, so the two unknown homology groups are zero.

(3) Finally, for $j \leqslant i$, we have

$$
0 \rightarrow H_{2 k}(A \cap B) \rightarrow \mathbb{Z} \oplus \mathbb{Z} \rightarrow \mathbb{Z} \rightarrow H_{2 k-1}(A \cap B) \rightarrow 0
$$

By Lemma 1, the central arrow is a surjective mapping, so

$$
H_{2 i}(A \cap B)=\mathbb{Z}, \quad H_{2 i-1}(A \cap B)=0 .
$$

Note that since $\mathcal{C}_{\pi, a}=A \cap B$ is a manifold, one can use the Poincaré duality and needs not analyze the third type of the sequences.

Example: the table for $n=7$, and $(\pi, a) \in W_{3}$ is completed like this: 


\begin{tabular}{|l|l|l|l|}
\hline $\mathrm{j}$ & $H_{*}\left(\mathcal{C}_{\pi, a}, \mathbb{Z}\right)$ & $H_{*}(A, \mathbb{Z}) \oplus H_{*}(B, \mathbb{Z})$ & $H_{*}(A \cup B, \mathbb{Z})$ \\
\hline 10 & 0 & 0 & $\mathbb{Z}$ \\
9 & $\mathbb{Z}$ & 0 & 0 \\
8 & 0 & 0 & $\mathbb{Z}$ \\
7 & $\mathbb{Z}$ & 0 & 0 \\
6 & 0 & $0 \oplus \mathbb{Z}$ & $\mathbb{Z}$ \\
5 & 0 & 0 & 0 \\
4 & 0 & $0 \oplus \mathbb{Z}$ & $\mathbb{Z}$ \\
3 & 0 & 0 & 0 \\
2 & $\mathbb{Z}$ & $\mathbb{Z} \oplus \mathbb{Z}$ & $\mathbb{Z}$ \\
1 & 0 & 0 & 0 \\
0 & $\mathbb{Z}$ & $\mathbb{Z} \oplus \mathbb{Z}$ & $\mathbb{Z}$ \\
\hline
\end{tabular}

Now let us prove (2) and (4). Set $A=\Phi^{-1}(\pi,(-\infty, a+\varepsilon])$ with some small $\varepsilon>0$. By Morse theory, $A$ has a homotopy type of a cell complex with even-dimensional cells only, one cell in each even dimension ranging from 0 to $i$. Set also $B=\Phi^{-1}(\pi,[a-\varepsilon, \infty))$. It also has one cell in each even dimension ranging from 0 to $2 n-4-i$.

The intersection $A \cap B$ is homotopy equivalent to $\mathcal{C}_{\pi, a}$, and a similar analysis of the Mayer-Vietoris sequence completes the proof.

Example: the table below depicts the case $n=6, a=0, i=4$.

\begin{tabular}{|l|l|l|l|}
\hline & $H\left(\mathcal{C}_{\pi, 0}, \mathbb{Z}\right)$ & $H(A, \mathbb{Z}) \oplus H(B, \mathbb{Z})$ & $H(A \cup B, \mathbb{Z})$ \\
\hline 8 & 0 & 0 & $\mathbb{Z}$ \\
7 & $\mathbb{Z}$ & 0 & 0 \\
6 & 0 & 0 & $\mathbb{Z}$ \\
5 & $\mathbb{Z}$ & 0 & 0 \\
4 & $\mathbb{Z}$ & $\mathbb{Z} \oplus \mathbb{Z}$ & $\mathbb{Z}$ \\
3 & 0 & 0 & 0 \\
2 & $\mathbb{Z}$ & $\mathbb{Z} \oplus \mathbb{Z}$ & $\mathbb{Z}$ \\
1 & 0 & 0 & 0 \\
0 & $\mathbb{Z}$ & $\mathbb{Z} \oplus \mathbb{Z}$ & $\mathbb{Z}$ \\
\hline
\end{tabular}

\section{DuAlity in the isoperimetric PROBlem}

The following theorem demonstrates equivalence of level foliations of $\mathcal{A}$ and $\mathcal{P}$.

Theorem 3. If $a>0$, there exists a level-preserving homeomorphisms

$$
\Psi_{+}:\left.\mathcal{C}_{a} \longrightarrow \mathcal{C}_{\pi}\right|_{\mathcal{A}>0}
$$

which sends Morse points of index $i$ to those of index $2 n-4-i$.

If $a<0$, the analogous homeomorphism

$$
\Psi_{-}:\left.\mathcal{C}_{a} \longrightarrow \mathcal{C}_{\pi}\right|_{\mathcal{A}<0}
$$

preserves Morse indices. 
Proof. Assume that $a>0$. We need to construct the horizontal maps in the following commutative diagram:

$$
\begin{aligned}
\mathcal{C}_{a} & \left.\cong \mathcal{C}_{\pi}\right|_{\mathcal{A}>0} \\
\downarrow \mathcal{P} & \downarrow \mathcal{A} \\
\mathbb{R}_{>0} & \cong \mathbb{R}_{>0} .
\end{aligned}
$$

Observe that $\Phi$ is a stratified submersion. The proof comes from "le rabattement de diagramme de Cerf", well known in singularity theory, and used e.g. in [12] and [10]. We rotate the vertical half line $\mathcal{P}=\pi$ with $\mathcal{A}>0$ to the horizontal position $\mathcal{A}=a$. An explicit map

$$
\mathcal{C}_{\pi} \cap\{\mathcal{A}>0\} \rightarrow \mathcal{C}_{a}
$$

can be obtained by scaling action, which gives a level preserving homeomorphism, see Figure 3, It follows that $\mathcal{P}$ and $-\mathcal{A}$ have the same Morse evolution.

Since $\mathcal{A}=0$ splits $\mathcal{C}_{\pi}$ into two symmetric pieces, the analogous statement applies for $\left.\mathcal{C}_{\pi}\right|_{\mathcal{A}<0}$

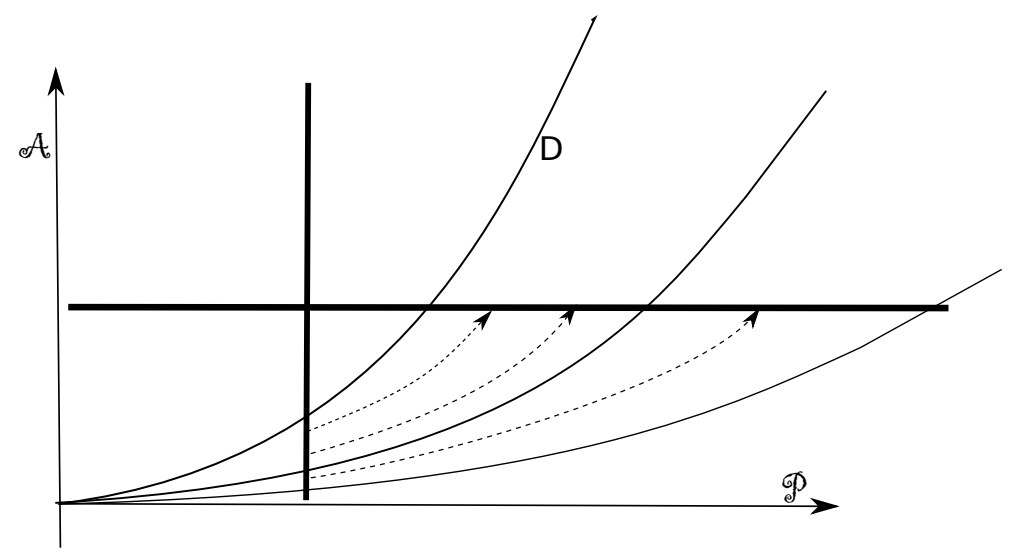

FIGURE 3. Rotating the half line to the horizontal position

The proof of Theorem 3 reveals the following general statement about 'kissing' surfaces:

Proposition 2. Let

$$
f:\left(\mathbb{R}^{n+1}, 0\right) \rightarrow(\mathbb{R}, 0) \quad \text { and } \quad g:\left(\mathbb{R}^{n+1}, 0\right) \rightarrow(\mathbb{R}, 0)
$$

be germs of two functions such that $X=f^{-1}(0)$ and $Y=g^{-1}(0)$ are smooth hypersurfaces tangent to each other at $(0,0)$. Assume that $\left.f\right|_{Y}$ has a Morse singularity of index $M$ at $(0,0)$. Then also $\left.g\right|_{X}$ has a Morse singularity at $(0,0)$, and its index $m$ satisfies

$$
\begin{cases}M+m=n, & \text { if } \nabla f \text { and } \nabla g \text { are codirected; } \\ M=m, & \text { if } \nabla f \text { and } \nabla g \text { are oppositely directed. }\end{cases}
$$

NB. This fact can be also demonstrated by analytic methods. It serves as the first important step in understanding the duality.

We immediately obtain the "dual" version of Theorem 2,

Theorem 4. For $a \neq 0$, the restriction $\left.\mathcal{P}\right|_{\mathcal{C}_{a}}$ is a perfect Morse function. 
(1) If $a>0$, the critical points of $\left.\mathcal{P}\right|_{\mathcal{C}_{a}}$ are exactly the regular stars with positive winding numbers. For each of the critical points, its Morse index satisfies

$$
m(P)+M(P)=2 n-4
$$

(2) If $a<0$, the critical points of $\left.\mathcal{P}\right|_{\mathcal{C}_{a}}$ are exactly the regular stars with negative winding numbers. For each of the critical points, its Morse index satisfies

$$
m(P)=M(P)
$$

In both cases $M(P)$ comes from Theorem 1 .

Another theorem follows directly.

Theorem 5. (1) The space $\mathcal{C}_{a}$ of polygons with prescribed area $a \neq 0$ is $a 2 n-4$ dimensional manifold and has a homotopy type of a cell complex with even cells only. The dimensions of the cells range from 0 to $\left[\frac{n+1}{2}\right] \cdot 2-4$, one cell in each even dimension.

(2) The space of polygons with zero area is homeomorphic to $\mathcal{C}_{\pi, 0} \times \mathbb{R}_{>0}$, so its homologies follow from Theorem 2.

Let us finish the paper by the concluding remark:

A similar duality approach is possible for any two functions $f$ and $g$, especially if both are homogeneous. In particular, one can think about sum of squared edge lengths versus area on the polygon space.

Another example is Coulomb energy and perimeter on polygon space (in this case critical points for $n=3$ and fixed perimeter are described in [6]. An interesting question is to extend the approach of the present paper to three or more functions.

\section{Appendix}

Proposition 3. The complete fold is a Morse point of the function $\mathcal{A}$ restricted to $\mathcal{C}_{\pi}$.

We may assume that the complete fold is $P^{o}=\left(p_{1}^{o}, \ldots, p_{n}^{o}\right)$ with $p_{1}^{o}=0$ such that $\vec{a}_{i}^{o}=$ $\vec{p}_{i}^{o} \vec{p}_{i+1}=(-1)^{i+1} \vec{a}$ for some non-zero vector $\vec{a}$. Let us coordinatize a neighborhood of $P^{o}$ by $\left(\vec{b}_{1}, \ldots, \vec{b}_{n-1}\right)$ where

$\vec{a}_{i}=\vec{p}_{i} \vec{p}_{i+1}=(-1)^{i+1} \vec{a}+\vec{b}_{i}, \quad i=1, \ldots, n-1$.

It is important that these coordinates describe the space of polygons with rotations and dilations not factored out. of $\mathcal{A}$.

Since the area vanishes at $P^{o}$, computing the 2 -jet of $\frac{\mathcal{A}}{\mathcal{P}^{2}}$ reduces to computing the 2 -jet

Now direct computations show that the Hessian matrix of $\frac{\mathcal{A}}{\mathcal{P}^{2}}$ has the following form: 


$$
\left(\begin{array}{cccccccccc}
0 & 0 & 0 & 1 & 0 & 0 & 0 & 0 & 0 & 0 \\
0 & 0 & -1 & 0 & 0 & 0 & 0 & 0 & 0 & 0 \\
0 & -1 & 0 & 0 & 0 & 1 & 0 & 0 & 0 & 0 \\
1 & 0 & 0 & 0 & -1 & 0 & 0 & 0 & 0 & 0 \\
0 & 0 & 0 & -1 & 0 & 0 & 0 & 1 & 0 & 0 \\
0 & 0 & 1 & 0 & 0 & 0 & -1 & 0 & 0 & 0 \\
0 & 0 & 0 & 0 & 0 & -1 & 0 & 0 & 0 & 1 \\
0 & 0 & 0 & 0 & 1 & 0 & 0 & 0 & -1 & 0 \\
0 & 0 & 0 & 0 & 0 & 0 & 0 & -1 & 0 & 0 \\
0 & 0 & 0 & 0 & 0 & 0 & 1 & 0 & 0 & 0
\end{array}\right)
$$

This is the matrix for $n=6$. In the general case we have a similar matrix $2(n-1) \times$ $2(n-1)$. It is sufficient to prove that its rank equals $2 n-4$ since two zero eigenvalues correspond to rotations and dilations. Eliminate the last two rows and the last to columns of the matrix. The resulted matrix is non-degenerate. Indeed, it is easy to conclude that its kernel vanishes.

\section{ACKNOWLEDGMENTS}

This work is supported by the RFBR grant 20-01-00070. It is our pleasure to acknowledge the hospitality and excellent working conditions of CIRM, Luminy, where this paper was initiated and completed as a 'research in pairs' project.

\section{REFERENCES}

[1] A. A. Agrachev, D. Pallaschke, and S. Scholtes, On Morse theory for piecewise smooth functions, J. Dynam. Control Systems, 3, No. 4, 449-469 (1997).

[2] A. Alaoui, A. Zeggar, Area and perimeter foliation on spaces of polygons, Graduate J. Math. 4 (2019), 1, 18-29.

[3] F. H. Clarke, Generalized gradients and applications, Trans. Amer. Math. Soc., 205, 247-262 (1975).

[4] J. Gordon, G. Panina, Y. Teplitskaya, Polygons with prescribed edge slopes: configuration space and extremal points of perimeter, arXiv:1712.00299v1, to appear in "Contributions to Algebra and Geometry."

[5] G. Giorgadze, G. Khimshiashvili, On area foliation of triangles, Bull. Georgian. Nath. Acad. Sci. 14 (2020), 1, 15-22.

[6] G. Khimshiashvili, G. Panina, D. Siersma, Equilibria of three constrained point charges, Journal of Geometry and Physics, 106 (2016) 42-50

[7] G. Khimshiashvili, G. Panina, D. Siersma, Extremal areas of polygons with fixed perimeter, to appear in Journal of Mathematical Sciences, available at http://www.pdmi.ras.ru/znsl/2019/v481/abs136.html

[8] K. Leichtweiss, Convex Geometry, Springer, 1980

[9] Khimshiashvili G., Panina G., Cyclic polygons are critical points of area. Zap. Nauchn. Sem. S.-Peterburg. Otdel. Mat. Inst. Steklov. (POMI), 2008, 360, 8, 238-245.

[10] D. Siersma, The monodromy of a series of hypersurface singularities, Comment. Math. Helv. 65 (1990), 2, 181-197.

[11] D. Mamaev, Oriented Area as a Morse Function on Configuration Spaces of Necklaces, arXiv:2001.02707

[12] M. Tibăr, Bouquet decomposition of the Milnor fibre. Topology 35 (1996), 1, 227-241.

[13] G. Panina, A. Zhukova, Morse index of a cyclic polygon,Cent. Eur. J. Math., 9(2) (2011), 364-377. 
[14] Zhukova A., Morse index of a cyclic polygon II, St. Petersburg Math. J. 24 (2013), 461-474.

G. Khimshiashvili: Ilia State University, Tbilisi; Gogikhim@yahoo.com; G. Panina: St. Petersburg Department of Steklov Mathematical Institute; St. Petersburg State University; Gaiane-Panina@rambler.ru; D. Siersma: Utrecht University, DePARTMENT OF MATHEMATiCS; D.SIERSMA@UU.NL 